\title{
Guía de Práctica Clínica: \\ Abordaje del paciente con lumbalgia crónica inespecífica
}

\author{
Approach to the patient with chronic non-specific low back pain.
}

\section{Introducción}

La lumbalgia es un problema frecuente en atención primaria y aunque sólo un pequeño porcentaje de los pacientes requerirá una consulta con un especialista, se trata del motivo de consulta más frecuente de los pacientes que acuden a las clínicas de dolor.

Se denomina lumbalgia aguda a la que dura hasta seis semanas de iniciado el cuadro, ya que la mayoría de estos episodios se autolimitan durante ese tiempo en forma espontánea o con mínimas intervenciones.

Por el contrario, la lumbalgia crónica (la que dura más de seis semanas) suele consumir muchos más recursos que la aguda $y$ es habitual que se la aborde con diferentes estrategias, muchas de ellas sin evidencia que la respalde.

\section{Objetivos de la guía}

Resumir las principales estrategias que hoy están recomendadas en la evaluación y tratamiento de los pacientes con lumbalgia inespecífica que persiste más allá de las seis semanas.

\section{La Guía}

Esta guía sólo aborda el manejo de los pacientes con lumbalgia crónica en quienes no se ha logrado detectar una "causa orgánica" que la explique.

La evaluación inicial de estos pacientes debería estar orientada a descartar síntomas o signos de infección, enfermedad oncológica secundaria, fractura, síndrome de cola de caballo, espondilitis anquilosante $\mathrm{y} / \mathrm{u}$ otras enfermedades inflamatorias, recomendándose tener presente la posibilidad de estos diagnósticos durante el tiempo que dure la evaluación y el tratamiento de estos pacientes. Si se sospecha alguno de estos cuadros, está recomendado solicitar una resonancia nuclear magnética (RNM) de la región de la columna involucrada.

El diagnóstico es primordialmente clínico y basado en la semiología del interrogatorio y el examen físico, no estando recomendado solicitar radiografías de columna lumbar sistemáticamente (en ausencia de sospecha de las condiciones clínicas descriptas previamente). Respecto de los pacientes con síndromes de lumbalgia crónica inespecífica, podría ser útil contar con una RNM en los pacientes que luego sean interconsultados con un traumatólogo que cuente con experiencia en patología del raquis.

\section{Puntos clave de la guía}

\section{Enfoque centrado en el paciente}

Propone considerar las preferencias y las necesidades individuales de los pacientes, siendo para esto esencial que se establezca una buena comunicación para que estos puedan elegir una vez que estén bien informados.

\section{Promoción del el auto-cuidado}

Tanto para el tratamiento como para la prevención de una futura recaída, se aconsejará a los pacientes que realicen ejercicios, que se mantengan físicamente activos y que lleven el estilo de vida más normal que puedan. Para promover el autocuidado son importantes el consejo y la educación. La educación incluirá información acerca de la naturaleza del dolor lumbar inespecífico, pudiendo también incluir material informativo, pero no siendo suficiente esta última opción como única estrategia terapéutica.

\section{Ofrecimiento de tratamiento analgésico}

El objetivo del tratamiento analgésico es aliviar el dolor para favorecer la actividad física. En cuanto al abordaje farmacológico la guía propone un manejo escalonado, recomendando comenzar con dosis regladas de paracetamol. Cuando este esquema sea insuficiente se puede ofrecer otros anti-inflamatorios no esteroideos (AINE) y/u opiáceos débiles (p. ej. codeína). Frente a esta última alternativa debería tenerse en cuenta el riesgo de dependencia y los efectos adversos que puedan ocasionar. Respecto de los opiáceos fuertes (p. ej. buprenorfina) se considerarán por un corto tiempo en quienes manifiesten dolor severo.

Para los mayores de 45 años que requieran uso de AINE, la guía recomienda prescribir un "protección de la enfermedad úlcero-péptica" con inhibidores de la bomba de protones (Ej. omeprazol).

Los antidepresivos tricíclicos se podrán considerar si la medicación antes mencionada fue insuficiente, comenzando con dosis bajas y aumentándolas hasta conseguir el efecto terapéutico, hasta la aparición de efectos adversos o hasta la llegada a la dosis máxima. Si bien una revisión sistemática encontró resultados contradictorios en cuanto al alivio del dolor y la recuperación de la funcionalidad, la guía considera que su costo es igual al del paracetamol y que no implica mayores efectos adversos, por lo que recomiendan su uso.

\section{Ofrecimiento de otras opciones terapéuticas}

Los autores recomiendan prescribir un programa de ejercicios, terapia manual y/o acupuntura; ofreciendo una opción diferente de la elegida inicialmente en los pacientes que no mejoran con la primera.

Los programas estructurados de ejercicio deberían incluir actividad aeróbica, fortalecimiento muscular, control postural y elongación, además de brindar instrucciones acerca de cómo moverse y como realizar ejercicios. Pueden implementarse en programas de hasta ocho sesiones a lo largo de doce semanas, que deben ser supervisados, y pueden ser individuales o grupales, con un máximo de diez integrantes por grupo (recomendación tipo A proveniente de un ensayo clínico de buen diseño metodológico)

La terapia manual puede implementarse como máximo en nueve sesiones a lo largo de 12 semanas y puede incluir manipulación espinal, movilización de la columna y masajes (recomendación tipo A proveniente de un ensayo clínico de buen diseño metodológico)

Respecto de la acupuntura, pueden ofrecerse hasta diez sesiones de la misma a lo largo de doce semanas (recomendación tipo A, proveniente de los resultados de una revisión sistemática)

La combinación de terapia manual junto con un programa de ejercicios es el tratamiento más costo/efectivo.

Pacientes con lumbalgia crónica refractaria: a los pacientes que ya hayan intentado las estrategias arriba expuestas y que aún tengan una gran discapacidad la guía recomienda prescribir un tratamiento psicológico con un abordaje cognitivo-conductual junto con un programa de ejercicios físicos (con una duración total aproximada de cien horas y una extensión de ocho semanas).

Si el paciente ya ha completado un esquema completo de tratamiento (incluido un programa físico y psicológico) y aún persiste con dolor lumbar severo, se recomienda interconsultarlo con un especialista, quien evaluara si es pertinente o no la opción quirúrgica. 


\section{Opciones no recomendadas por esta guía}

Los autores recomiendan no prescribir la colocación de soportes en la espalda (p. ej. fajas o corsets) no ofrecer inhibidores selectivos de la recaptación de serotonina (IRSS) no efectuar tracción de la columna, no administrar sustancias terapéuticas en la espalda (ej. infiltraciones) y no usar terapia con láser, ultrasonido, estimulación eléctrica transcutanea (en inglés TENS) ni terapia termoeléctrica intra-discal.

\section{Comentario}

Un $80 \%$ de la población sufre lumbalgia en algún momento de su vida ${ }^{1}$, el 20 al $25 \%$ de los pacientes con lumbalgia baja se ausentan de su trabajo por tiempo prolongado².

El abordaje apropiado de los pacientes con lumbalgia crónica inespecífica demanda, además de un cuerpo de conocimiento integral por parte de los profesionales actuantes, un equipo interdisciplinario que incluya enfermeros, psicólogos, osteópatas, kinesiólogos, quiroprácitos, profesores de educación física, cirujanos traumatólogos, así como una estructura edilicia donde se pueda ofrecer diferentes programas de rehabilitación. En el desarrollo de la guía que hemos resumido se especifica a los destinatarios de este material educativo y se enumeran las causas de lumbalgia específica que hay que descartar antes de incluir a los pacientes en abordajes conservadores, los diagnósticos diferenciales hay que tener en cuenta, los estudios de imágenes que hay que pedir y en que momento, conceptos sobre el consejo y la educación para los pacientes, la medicación a utilizar, las diferentes opciones terapéuticas disponibles; y el mejor momento para realizar intervenciones intensivas y/o derivar al paciente al especialista.

Si bien propone una evaluación esquematizada y clara de cómo evaluar a los pacientes con lumbalgia de más de seis semanas de evolución, termina siendo confusa en algunos aspectos del manejo. Consideramos que no es clara cuando recomienda avanzar con diferentes programas ante un paciente con una mala evolución al tratamiento, en las recomendaciones con respecto a las intervenciones psicosociales y respecto del uso de antidepresivos. Esto es entendible ya que se trata de una condición clínica para la que todavía no existe la documentación de un estándar de tratamiento claramente superior a los demás, lo que conduce a que ganen espacio las conductas basadas en la experiencia del equipo tratante. Por ejemplo, a pesar de que no se ha encontrado evidencia que sustente el uso de antidepresivos tricíclicos -una revisión sistemática sobre el tema arrojó resultados contradictorios- los autores de la guía igualmente aconsejan su uso (recomendación de expertos).

Otro concepto abordado por los autores y que consideramos que vale la pena discutir es el de lumbalgia "sin causa orgánica". Muchas veces se rotula a un paciente con esta etiqueta cuando no se le ha podido documentar a través del interrogatorio, el examen físico o exámenes complementarios alguna causa o mecanismo que explique el síntoma o su persistencia. Sin embargo, consideramos que este calificación es altamente dependiente del momento histórico en el que sucede la consulta, del paradigma en el cual trabaja el equipo tratante -“...si no lo encuentro con las maniobras del examen físico que yo se hacer (p.ej. Lasssegue) o si no lo veo en la resonancia, no hay enfermedad orgánica..."- de su habilidad semiológica y/o del tiempo dedicado en las consulta para intentar establecer dicha causalidad o mecanismo de perpetuación del dolor. Por ejemplo, en muchos pacientes que habían sido clasificados inicialmente (a veces durante años) como pertenecientes a la población que sufre una lumbalgia de causa "no orgánica" luego se constató que tenían alguna disfunción miofascial ${ }^{54,5}$, o tendinosa y tuvieron buena respuesta a tratamientos enfocados a dicha re-calificación sindromática.

\section{Conclusiones de los comentadores}

De todos modos y ante la contraposición entre la enorme cantidad de alternativas terapéuticas para quienes sufren esta condición clínica y la escasez de los recursos, celebramos esta nueva guía de práctica clínica que nos ayuda a clasificar la evidencia disponible, a priorizar las intervenciones disponibles y a establecer un pautas para algún eventual protocolo racional de manejo escalonado de estos pacientes.

Ignacio Vicente Lago y Sergio Terrasa [ Senvicio de Medicina Familiar del Hospital Italiano de Buenos Aires ] sergio.terrasa@ hospitalitaliano.org.ar

Vicente Lago I. Guía de Práctica Clínica para el manejo de la lumbalgia crónica inespecífica. Comentado de: Early management of persistent nonspecific low back pain. NICE Guideline National Institute for Health and Clinical Excellence. May 2009. Disponible en Internet: www.nice.org.uk/CG88 (último acceso 05/05/10).

\section{Referencias}

1. Grupo de Trabajo Multidisciplinar para la elaboración de una Guía Clínica para la Evaluación y Tratamiento del Dolor Miofascial. Síndrome del dolor miofascial. 2007 IPSEN PHARMA, S.A. 2. Lambeek L y col. Randomised controlled trial of integrated care to reduce disability from chronic low back pain in working and private life. BMJ. 2010 Mar 16;340:c1035. doi:

10.1136/bmj.c1035.

4. Skootsky $S$ y col. Prevalence of myofascial pain in general internal medicine practice. West J Med. 1989;151(2):157-60.

5. Simons, David G. Travell \& Simons' myofascial pain and dysfunction : the trigger point manual / David G. Simons, Janet G. Travell. Lois S. Simons ; illustrations by Barbara D. Cummings, with contributions by Diane Abeloff and Jason Lee. 2nd ed.1999 Williams \& Wilkins. 2002. Part 5. Torso Pain. Chapter 41 . Overview of Torso Region. Pag. 801.

Vale destacar la serie de pacientes descripta por Rosomoff en 1990, que luego de examinar a 283 pacientes que habían sido rotulados de padecer "dolor crónico benigno intratable" encontró puntos gatillos de dolor miofascial en más del $85 \%$.

${ }^{\S}$ La disfunción miofascial explicaría el 20 al 30\% del total de los dolores osteomusculares que sufren pacientes del ámbito de la atención primaria y más del 70 ú $80 \%$ de los individuos seleccionados en servicios de reumatología o clínicas de dolor. En los pacientes con lumbalgia por disfunción miofascial, el músculo cuadrado lumbar es el más frecuentemente involucrado $(41 \%)$ seguido por el glúteo medio, el psoas-ilíaco y el piramidal. 